\title{
A CHARACTERIZATION OF IRREDUCIBLE SETS MODULO LEFT-LINEAR TERM REWRITING SYSTEMS BY TREE AUTOMATA
}

Z. Fülöp and $S$. Vágvölgyi Research Group on Theory of Automata Hungarian Academy of Sciences H-6720 Szeged, Somogyi u. 7., Hungary

J. H. Gallier and R. V. Book showed that the set of irreducible terms modulo a left-linear term rewriting system can be recognized by a deterministic bottom-up tree automaton. However, this device is too general to characterize irreducible sets: there are recognizable term sets which cannot be the set of irreducible terms modulo any term rewriting system. We introduce the class of one-state deterministic top-down tree automata with prefix look-ahead, which exactly recognizes the class of irreducible terms modulo left-linear term rewriting systems.

A one-state deterministic top-down tree automaton with prefix look-ahead is a system $A=(\Sigma, T, P)$, where

(a) $\Sigma$ is a ranked alphabet,

(b) $q$ is the state of $A$,

(c) $P$ is a finite set of rules of the form

$\langle q \sigma \rightarrow(q, \ldots, q) ; L\rangle$ with $m \geq 0, \sigma \in \Sigma_{m}$, where $L=t T_{\Sigma}=\left\{t\left(t_{1}, \ldots, t_{n}\right) \mid t_{i} \in T_{\Sigma}\right.$ for $I \leq i \leq n$ ) for some linear tree $t \in T_{\Sigma}\left(x_{n}\right)$. Moreover, for any different rules $\left\langle q \sigma \rightarrow(q, \ldots q) ; L_{1}\right\rangle$ and $\left\langle q \sigma+(q, \ldots, q), L_{2}\right\rangle, L_{1} n_{L_{2}}=\varnothing$ holds. The tree language recognized by $A$ is $L(A)=\left\{t \in T_{\Sigma} \mid q(t) \stackrel{*}{\Rightarrow} t\right\}$, where $\stackrel{*}{\Rightarrow}$ is the reflexive-transitive closure of defined as follows: for $u, v \in T_{\Sigma}\left(q T_{\Sigma}\right), u \rightarrow v$ if and only if

(1) $u=c\left(q\left(\sigma\left(t_{1}, \ldots, t_{m}\right)\right)\right)$ for some $\left.c \in T_{\Sigma}\left(t x_{1}\right)\right)$, $x_{1}$ occurs once in $c, m \geq 0, \sigma \in \Sigma_{m}$ and $t_{1}, \ldots, t_{m} \in T_{\Sigma}$, (ii) $\mathrm{v}=\mathrm{c}\left(\sigma\left(\mathrm{q}\left(\mathrm{t}_{1}\right), \ldots, \mathrm{q}\left(\mathrm{t}_{\mathrm{m}}\right)\right)\right)$,

(iii) the rule $\langle q \sigma+(q, \ldots, q)$; $L\rangle$ is in $P$,

(iv) $\sigma\left(t_{1}, \ldots, t_{m}\right) \in I$.

Our main result is the following theorem.

Theorem: For any set $T$ of terms, $T$ is the set of irreducible terms with respect to a left-linear term rewriting system if and only if $T$ can be recognized by a one-state deterministic top-down tree automaton with prefix look-ahead. 